\title{
Effect of tip speed ratio of rear stage impeller on the performance of contra-rotating wind turbine
}

\author{
Zhiqiang $\mathrm{LI}^{1, \mathrm{a}}$, Zhiming $\mathrm{LI}^{2, \mathrm{~b}}$ * \\ ${ }^{1}$ National Key Laboratory of Science and Technology on Aero-Engine Aero-thermodynamics, \\ School of Energy Power Engineering, Beihang University, Beijing 100191, China \\ ${ }^{2}$ National Key Laboratory of Science and Technology on Aero-Engine Aero-thermodynamics, \\ School of Energy Power Engineering, Beihang University, Beijing 100191, China \\ alizhiqiang@buaa.edu.cn, 'lizhimingqq1225@126.com
}

\begin{abstract}
Keywords: wind power; contra-rotating wind turbine; tip speed ratio; numerical simulation.
Abstract. Based on the experimental research and numerical calculation of the Contra-Rotating Wind Turbine (CRWT) by using the technique of the aero-engine and propeller, the effect of the tip speed ratio of the rear stage on the performance of the contra-rotating wind turbine is studied by using numerical simulation method in this paper. The computational results show that there is an optimal tip speed ratio on the rear stage of the contra-rotating wind turbine under medium wind speed in order to achieve the highest output power. Below the optimal value, boundary layer separation of the rotor blade is serious, output power is lower, and output torque is larger; above the optimal value, the opposite situation occurs. Through analyzing the computational results, the optimal value of tip speed ratio of rear stage impeller is 1.667 .
\end{abstract}

\section{Introduction}

The wind energy is one kind of clean, renewable energy sources and the reserves is rich. To protect the environment and save the fossil fuel, many countries positively are developing the wind power to generate electricity. And expanding the scale of the using wind energy is also becoming an important trend in the current $[1,2]$. Because of the limitation of the wind energy sources, most of the wind power plants are located in the areas which the wind energy is rich. But along with the urban development, the wind energy is also becoming rich, for example, the corridors among the building groups and the rooftops of the high buildings. For utility of the urban wind energy, the miniaturization of the wind turbine must be considered. To increase the performance, huge size rotor with 3 blades is the most popular configuration, while with the decrease of the size, the performance will also be reduced.

To solve the conflict between the size and the performance, the contra-rotating wind turbine (CRWT) that has two rotors at the same axis and rotating in the opposite direction at the same axis is proposed by the authors and the feasibility and advantage in power extraction is proved by experiments [3]. Compared with the single rotor wind turbine, the output power of CRWT was obvious increased. And the research results also prove CRWT has a great potential to solve the conflict between the size and the performance.

The performance of CRWT has been considered in some publications. M.Curtis Rector et al. analyzed the structure of contra-rotating wind turbine, their research results show that: when the tip speed ratio is very big, counter-rotating wind turbine does not have much more advantages than traditional wind turbine with single impeller; but when the tip speed is very small, especially the tip speed ratio is less than 3, counter-rotating wind turbine can significantly improve the utilization rate of wind; they also calculated the wind power utilization rate of small counter-rotating wind turbine is nearly $30 \%$ [4].

Based on the previous research, to optimize design of CRWT with the structure and aerodynamic performance of the wind turbine, the effect of the tip speed ratio of the rear stage on the performance of the contra-rotating wind turbine is studied by using numerical simulation method in this paper. 


\section{Numerical Simulation}

Modeling of Wind Turbine. In this paper, the optimization design scheme is put forward on the basis of the experiment with principle prototype in air wind tunnel, so the model parameters of wind turbine is limited by principle prototype and wind tunnel measurement in mathematical modeling process [5]. Considering the actual environment wind speed and the minimum effective experimental wind speed of wind tunnel in our laboratory, the wind speed is set for $10 \mathrm{~m} / \mathrm{s}$ in the numerical simulation. The related geometrical parameters of wind turbine as shown in table 1. The wind turbine model is shown in figure 1 .

Table 1 Basic data of Contra-rotating impellers

\begin{tabular}{|c|c|c|c|c|c|}
\hline Stage & Airfoil & $\begin{array}{l}\text { Impeller diameter } \\
-D[\mathrm{~mm}]\end{array}$ & $\begin{array}{l}\text { Chord length } \\
-L[\mathrm{~mm}]\end{array}$ & $\begin{array}{c}\text { Angle of attack } \\
-\alpha\left[^{\circ}\right]\end{array}$ & $\begin{array}{c}\text { Impeller } \\
\text { spacing- } d[\mathrm{~mm}]\end{array}$ \\
\hline Front & \multirow{2}{*}{$\begin{array}{c}\text { NACA } \\
4418\end{array}$} & 400 & 40 & 15 & \multirow{2}{*}{80} \\
\hline Rear & & 500 & 50 & 20 & \\
\hline
\end{tabular}

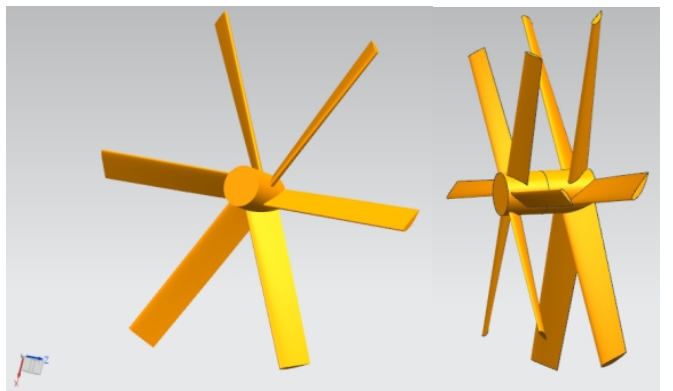

Figure 1Wind turbine rotor model

Mesh Generation. During the calculation process, it is important to specify the computational domain, that is, the artificially imposed boundary constraints of the flow of wind turbine. Therefore, the selection of computational domain is directly related to the effectiveness of numerical simulation. In this study, the cylindrical computational domain is choosed. Because the structure of rotor blade is complicated, unstructured grid is used [6]. Considering the quality of the mesh directly affects accuracy of the results, the grid surrounding the wind turbine is refined, while the size of the grid on far-field will turn bigger. The overall grid node number is 207248, total number of grid is 1202678 . Figure 2 shows the grid conditions near the two blades.

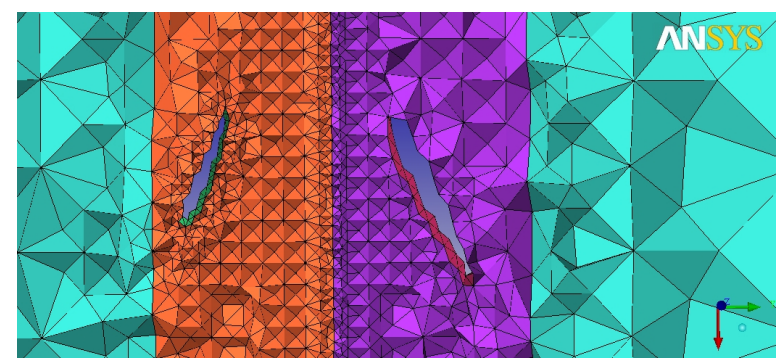

Figure 2Grid display near the blades

Calculate Solution. Taking Fluent14.0 as solver, this paper selected three equation model $k-k l-\omega$ to simulate the flow around the wind turbine. Compared with the conventional two equation turbulence model, this model can more accurately simulate the transition flow from laminar to turbulent flow, and has more computing capability for inlet internal complex flow, so it can be applied to simulate the transition of the flow field between the front rotor and the rear rotor. Fluid is incompressible air, solution is based on $\mathrm{N}-\mathrm{S}$ equations of three dimensional separation implicit method, 
and SIMPLEC algorithm is used in coupling of the pressure and velocity. For the convection items, such as momentum and kinetic energy, the discrete scheme with second order upwind scheme is adopted, while central difference format is used for the dissipative item [7]. Convergence errors of all equations are set to 0.00001 .

During the calculation, the velocity inlet and pressure outlet are used, and the outlet pressure is set as the standard atmospheric pressure. The wall is defined as the non slip condition. The area between two stages is defined as the rotating flow. The flow field information is transmitted and coupled with the Multiple Reference Frame Model (MRF).

\section{Results and Analysis}

Validation of Numerical Calculation Method. In order to test the $k-k l-\omega$ model can effectively simulate the aerodynamic performance of the twisted blade contra-rotating wind turbine, the numerical calculation method is verified by the experimental results of the initial stage [3]. The power output of straight blades contra-rotating wind turbine with different tip speed ratios is shown Fig.3. It can be seen that the simulation results agree well with the test results, so the numerical simulation method selected s can effectively reflect the influence caused by tip speed ratio on the properties of twisted blades rotating wind turbine.

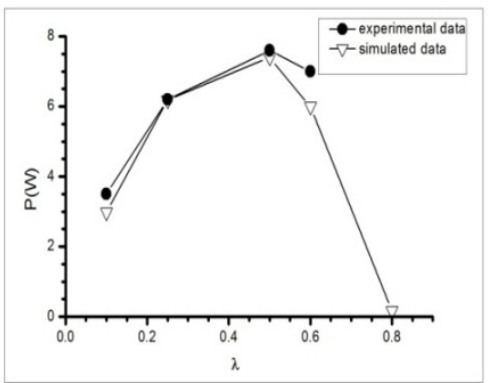

Figure 3 Power curve under different tip speed ratio

Relationship between Tip Speed Ratio of Rear Stage and Output Power. During the simulation, the distance between front and rear stage is kept as $60 \mathrm{~mm}$, the tip speed ratio of front stage $\lambda$ is 0.25 , and the wind speed $V_{u}$ is defined as $15 \mathrm{~m} / \mathrm{s}$. In order to study the relationship between wind turbine aerodynamic performance and the tip speed ratio of rear stage, four schemes are set for the tip speed ratio the tip speed ratio of rear stage, i.e. $0.167,0.250,0.333,0.500,0.833$. The computational results of the relationship between output power and tip speed ratio are shown in Figs. 4, 5, 6. Fig. 7 shows the relationship between output torque and tip speed ratio.

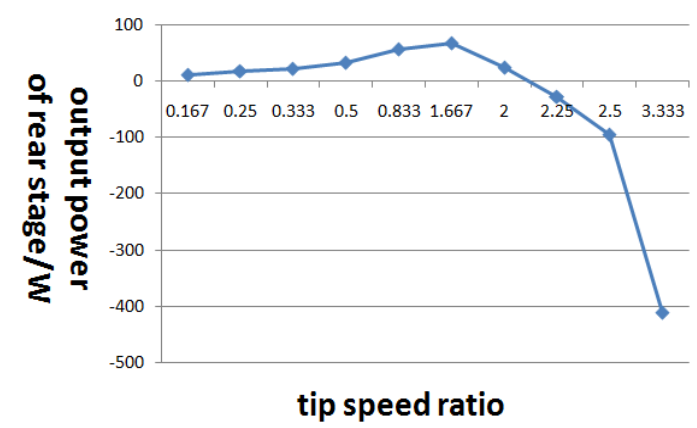

Figure 4 output power of rear stage - tip speed ratio

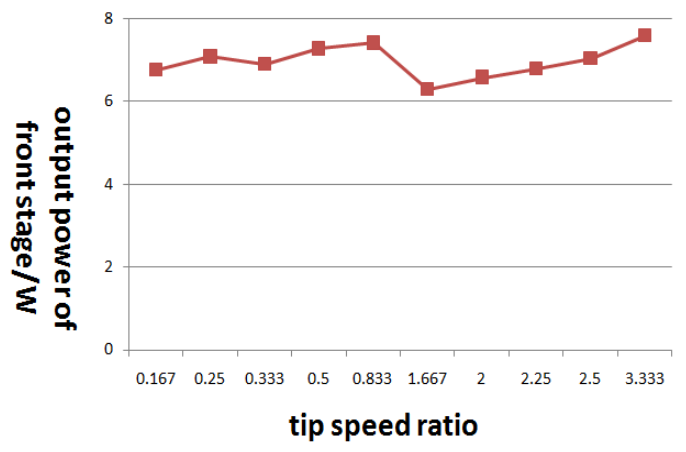

Figure 5 output power of front stage - tip speed ratio 


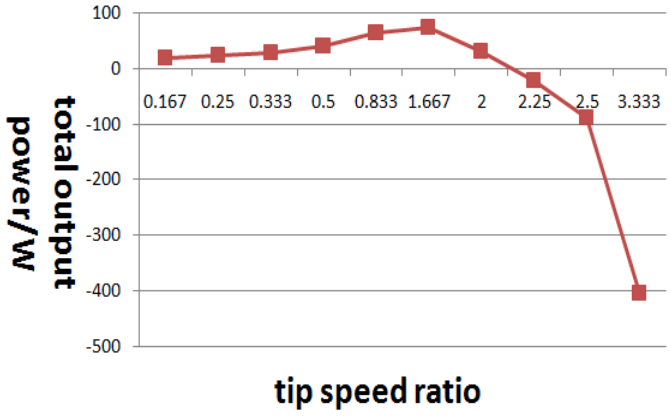

Figure 6 total output power - tip speed ratio

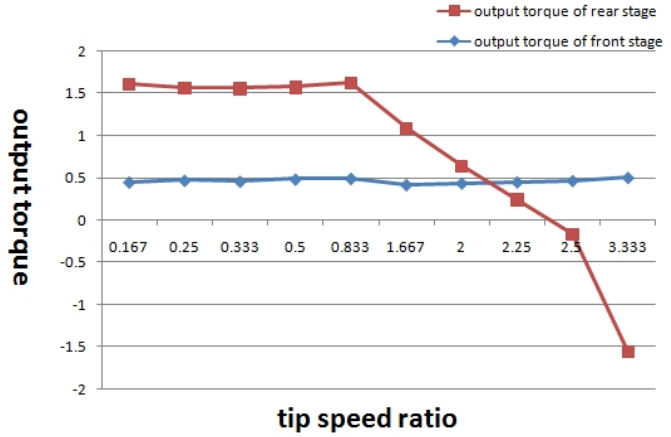

Figure 7 output torque of rear stage - tip speed ratio

As can be seen from the figure 4 to figure 6 , the output power of front stage is almost not influenced by the tip speed ratio of rear stage, and it is consistent with the results of previous studies [8]. Here is verified the validity of the numerical simulation again. However, the output power of rear stage is seriously influenced by the tip speed ratio of rear stage. When the tip speed ratio is 1.667, output power of rear stage is the biggest: $P_{\text {opt }}=73.3 \mathrm{~W}$, and the output torque is very small. With the increase of the tip speed ratio, when the tip speed ratio is greater than 2.1 , output torque will be negative, it actually does not exist. In the condition of low torque, requirements on the performance of generator is very high, current technology is still not meet such requirements. Therefore, when we design of contra-rotating wind turbine, Must be on the basis of both "tip speed ratio - torque" and "tip speed ratio - power". In this experiment, it can be broadly recognized the best tip speed ratio of the rear stage $\lambda_{\text {opt }}=1.667$, we can calculate the best power coefficient :

$$
C_{p}=\frac{P_{\mathrm{opt}}}{\frac{1}{2} \rho \pi R^{2} V_{u}^{3}}=\frac{73.3}{\frac{1}{2} \times 1.205 \times 3.14 \times 0.25^{2} \times 15^{3}}=18.37 \%
$$

\section{Conclusion}

Based on the previous research, to optimize design of CRWT with the structure and aerodynamic performance of the wind turbine, the effect of the tip speed ratio of the rear stage on the performance of the contra-rotating wind turbine is studied by using numerical simulation method in this paper. The computational results show the optimal tip speed ratio of rear stage at the medium wind speed level. When the tip speed ratio is low, output power of wind turbine is small, but output torque is big. However, when the tip speed ratio is high, the results are opposite. As a results, under a certain wind speed, there is an optimal value of the tip speed ratio of the contra-rotating wind turbine. In this study, when the tip speed ratio of rear stage is 1.667 , the output power is the biggest.

\section{References}

[1] Shaoyu Su.The design and operation maintenance of the generator with wind power[M].Beijing:China electric power press,2003.(in Chinese)

[2] Qingyi Li .Small wind turbine design[M].Mechanical industry press, 1986.(in Chinese)

[3] Zhiqiang Li, Yong Wang, Yusheng Zhang. Experimental Study on Interaction of the Counter-Rotating Rotors in a Wind Turbine [C]. Proceedings 2011 international conference on Materials for Renewable Energy \& Environment, vol.01 Shanghai China, May, 2011, IEEE press, pp570-574 
[4] M. Curtis Rector, Kenneth D. Visser. Aerodynamic Design of a Small Contra-Rotating HAWT[A]. 45th AIAA Aerospace Sciences Meeting and Exhibit, AIAA-2007-1371, Reno, Nevada, January 8-11, 2007

[5] Zhiqiang Li, Yusheng Zhang.Basic research on contra-rotating wind turbine with double leaf blade[D].Beijing:Beihang University,2010.(in Chinese)

[6] Bingbing Ji,Jinping Chen.ANSYS ICEM CFD Meshing technology example explanation[M].China water conservancy and hydropower press,2012.(in Chinese)

[7] MatthieuDubosc,NicolsaTantot,PhilippeBeaumier,etal.A method for predicting contra rotating propellers off-design performance[C].Proceedings of ASME Turbo Expo 2014:Turbine Technical Conference and Exposition,June 16-20,2014,Dusseldorf,Germany,2014,16(6).

[8] Sørensen N N, Michelsen J A, Schreck S. Navier-Stokes predictions of the NREL phase VI rotor in the NASA Ames $80 \mathrm{ft} \times 120 \mathrm{ft}$ wind tunnel[J]. Wind Energy, 2002, 5(2- 3): 151-169. 